\title{
LOW POWER MEASUREMENTS ON AN AGS INJECTION KICKER MAGNET
}

\author{
M.J. Barnes ${ }^{\#}$, G.D. Wait, TRIUMF, Vancouver, B.C., Canada
}

\section{Abstract}

The present AGS injection kickers at A5 location were designed for $1.5 \mathrm{GeV}$ proton injection. Recent high intensity runs have pushed the transfer kinetic energy to $1.94 \mathrm{GeV}$, but with an imperfect matching in transverse phase space. Space charge forces result in both fast and slow beam size growth and beam loss as the size exceeds the AGS aperture. An increase in the AGS injection energy to $2 \mathrm{GeV}$ with adequate kick strength would greatly reduce the beam losses, making it possible to increase the intensity from $70 \mathrm{TP}\left(70 \cdot 10^{\wedge} 12\right.$ protons/s) to 100 TP. R\&D studies have been undertaken by TRIUMF, in collaboration with BNL, to design two new kicker magnets for the AGS A10 location to provide an additional kick of $1.5 \mathrm{mrad}$ to $2 \mathrm{GeV}$ protons. TRIUMF has designed a $12.5 \Omega$ transmission line kicker magnet with rise and fall times of $100 \mathrm{~ns}, 3 \%$ to $97 \%$, and field uniformity of $\pm 1 \%$ over $85 \%$ of the aperture, powered by matched $12.5 \Omega$ pulse-forming lines. This paper describes the results of detailed capacitance and inductance measurements, on the prototype magnet, and compares these with predictions from electromagnetic simulations.

\section{INTRODUCTION}

The design for the Alternating Gradient Synchrotron (AGS) A10, 11 cell, transmission line kicker magnets [1], is based on a TRIUMF prototype 10-cell kicker magnet $[2,3]$, which was itself based on designs originated at CERN [4]. The transmission line type kicker (Fig. 1) consists of ferrite $\mathrm{C}$-core sections sandwiched between high voltage (HV) capacitance plates. One C-core ferrite, together with its ground and HV capacitance plates, is termed a cell. Each cell conceptually begins and ends in the middle of the HV capacitance plates. Striplines, at the input and output of the kicker, have an impedance as close as possible to that of the kicker magnet.

Table 1: Existing A5 and proposed A10 kicker system parameters for $2 \mathrm{GeV}$ protons

\begin{tabular}{|l|l|l|}
\hline Parameter & Existing A5 & Proposed A10 \\
\hline Deflection angle & $2.48 \mathrm{mrad}$ & $1.5 \mathrm{mrad}$ \\
\hline Gap height & $57.15 \mathrm{~mm}$ & $81 \mathrm{~mm}$ \\
\hline Gap width & $127 \mathrm{~mm}$ & $137 \mathrm{~mm}$ \\
\hline Rise-time 3\% to 97\% & $140 \mathrm{~ns}$ & $100 \mathrm{~ns}$ \\
\hline Fall time 97\% to 3\% & $140 \mathrm{~ns}$ & $100 \mathrm{~ns}$ \\
\hline Flat-top Duration & 0 to $2250 \mathrm{~ns}$ & 0 to $1650 \mathrm{~ns}$ \\
\hline Number of magnets & 3 & 2 \\
\hline Magnetic field & $24.2 \mathrm{mT}$ & $19.1 \mathrm{mT}$ \\
\hline Current & $1100 \mathrm{~A}$ & $1235 \mathrm{~A}$ \\
\hline PFL Voltage & $32.5 \mathrm{kV}$ & $30.9 \mathrm{kV}$ \\
\hline Magnet type & Lumped & Transmission line \\
\hline
\end{tabular}

\# Now employed at CERN, Switzerland.

Work supported by a contribution from the Canada Foundation for Innovation.

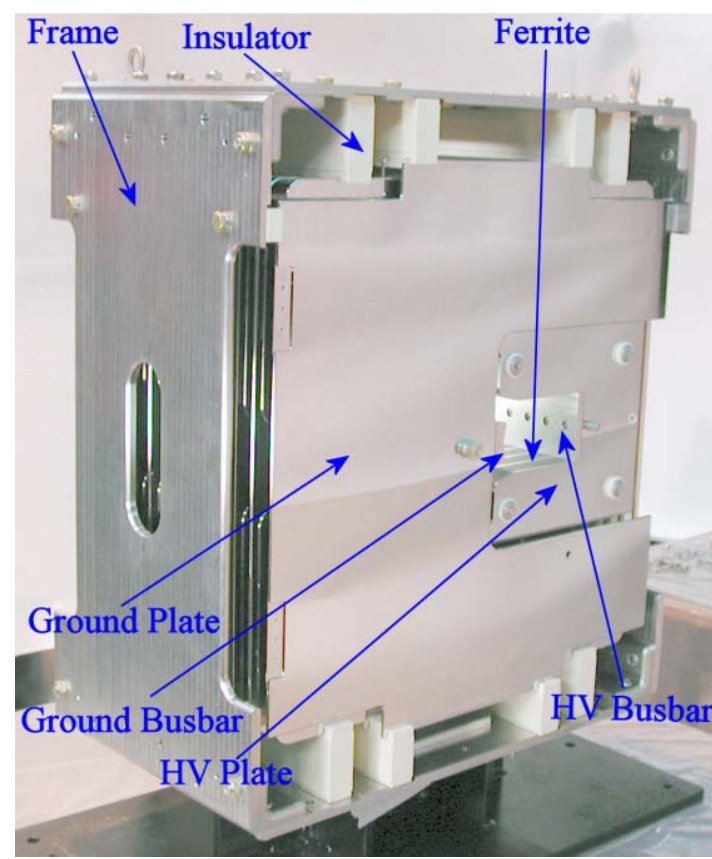

Figure 1: Prototype AGS A10 kicker magnet.

\section{DESIGN CONSIDERATIONS}

\section{General}

The rise-time of the magnetic field is dependent upon the rise-time of the input pulse, the delay of each cell $(\sqrt{L \cdot C})$, where $L$ and $C$ are the inductance and capacitance per cell of the kicker magnet, respectively, and the number of cells. To achieve rise and fall times of $100 \mathrm{~ns}$ (Table 1) two 11 cell kicker magnets are used, rather than one 22 cell magnet, each driven by its own pulse generator. The characteristic impedance $(Z)$ of a cell is given by Eq. 1 .

$$
Z=\sqrt{L / C}
$$

Impedance mismatches result in reflections which cause field ripple and potentially reduce the life of the thyratron switches. Therefore a carefully matched high bandwidth system is needed to obtain the required pulse response. Hence each of the two proposed A10 systems is composed of a Pulse Forming Line (PFL) and a travelling wave magnet, connected by a matched transmission line and terminated by a matched resistor (Fig. 2).

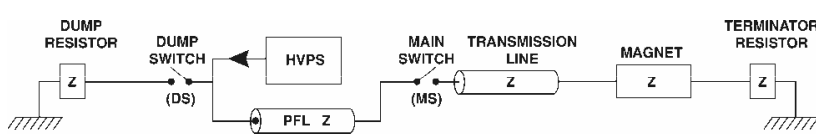

Figure 2: Schematic circuit of one kicker magnet system.

To obtain a compact and cost efficient design a low system impedance of $12.5 \Omega$ is chosen. Fig. 2 gives a circuit schematic for one of the kicker systems. 


\section{Simulations}

The magnetic and electric fields associated with the aperture of the prototype A10 kicker magnet have been studied using $2 \mathrm{D}$ and $3 \mathrm{D}$ electromagnetic codes: the magnetic field simulations included the effect of eddy currents [5]. The shape of the aperture was optimized to achieve a design goal of $\pm 3 \%$ deflection uniformity, for protons, throughout $90 \%$ of the aperture cross-sectional area. The predicted inductance of a central cell of the optimized geometry is $62.4 \mathrm{nH}[5]$. To achieve $12.5 \Omega$ characteristic impedance, the required capacitance is therefore $399.2 \mathrm{pF}$ per cell.

The capacitance of each cell is determined by the geometries of both the HV and ground plates. However edge effects of the plate result in a reasonable fraction of the capacitance of a cell, and thus the 3D code Coulomb [6] was used to predict the cell capacitance and finalize the geometry of the plates. The cell length is $30.06 \mathrm{~mm}$ and the thickness of each plate is modelled as $4.763 \mathrm{~mm}$, which results in a plate separation of $10.267 \mathrm{~mm}$. The selected geometry of the HV and ground plates gave a predicted central cell capacitance of $396.3 \mathrm{pF}$ [7], which is within $0.73 \%$ of the $399.2 \mathrm{pF}$ required for $12.5 \Omega$. The predicted edge effect capacitance for this plate geometry is $6.5 \mathrm{pF} / \mathrm{m}$.

Fringe fields result in an appreciable increase in inductance per cell towards the ends of a kicker $[3,5]$. Hence in order to obtain realistic inductance predictions the 3D eddy current code Elektra has been used to simulate the kicker magnet [5]. Elektra has successfully been applied, previously, to accurately predict the cell inductance of a transmission line type kicker magnet [3].

\section{MEASUREMENTS}

\section{Capacitance}

The thickness of the capacitor plates is less than expected: the measured thickness is between $4.56 \mathrm{~mm}$ and $4.59 \mathrm{~mm}$, which gives an average plate separation of $10.455 \mathrm{~mm}$, for a cell length of $30.06 \mathrm{~mm}$. Scaling the cell capacitance, from the predicted value of $396.3 \mathrm{pF}$, with plate separation $(10.455 \mathrm{~mm}$, rather than the expected $10.267 \mathrm{~mm}$ ), gives a cell capacitance of $389.2 \mathrm{pF}$.

The measured capacitance of the AGS magnet, using a Fluke PM6306 LCR meter [8], is in the range 4711.0pF to $4711.3 \mathrm{pF}$ : this is the capacitance for 12 sets of capacitor plates (11 cells). A frequency of $50 \mathrm{kHz}$ was chosen for this capacitance measurement because the specified basic measurement accuracy of the PM6306 meter is best and is $\pm 0.1 \% \pm 1$ digit (i.e. $\pm 4.7 \mathrm{pF} \pm 0.1 \mathrm{pF}=> \pm 4.8 \mathrm{pF}$ ) at this frequency [8]: this corresponds to $\pm 0.4 \mathrm{pF}$ per cell.

The Coulomb 3D simulations predict that an end cell has a capacitance $9 \mathrm{pF}$ greater than a central cell. Hence, correcting the measured capacitance for predicted end effects, results in an average capacitance of $391.1 \mathrm{pF} \pm 0.4 \mathrm{pF}$ per central cell. This is within $0.5 \%$ of the theoretical value of $389.2 \mathrm{pF}$.

\section{Inductance}

To determine inductance per cell of the A10 kicker magnet, it was driven with a low voltage pulse with a $7 \mathrm{~ns}$ rise-time. Fig. 3 shows the propagation of the pulse through each of the 11 cells of the kicker. A high impedance low-capacitance probe was used for measuring voltage on each of the HV capacitance plates: the kicker was driven from a $\sim 12.5 \Omega$ source and terminated with a $12.67 \Omega$ resistor. The 'flat-top' oscillations in Fig. 3 are damped out by an elapsed time of $\sim 450 \mathrm{~ns}$. These oscillations are excited by the fast $(7 \mathrm{~ns})$ rise-time of the input pulse; the rise-time of the actual HV pulse ( $\sim 30 \mathrm{~ns})$ would not excite such severe oscillations.

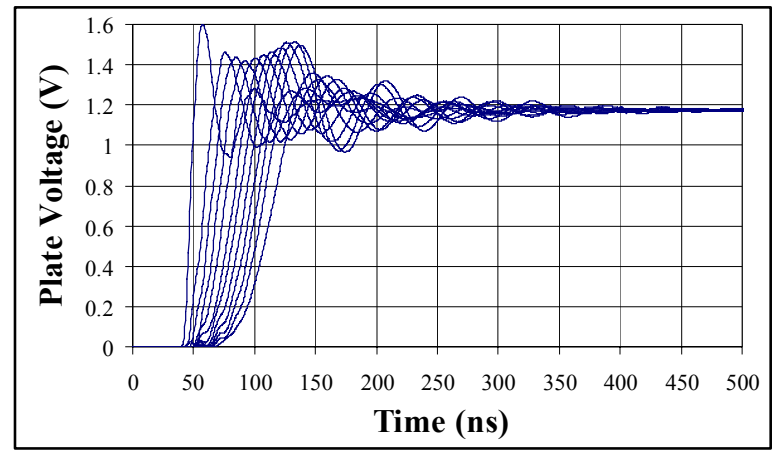

Figure 3: Pulse propagation through the prototype kicker.

The inductance of a cell was determined from the measured pulse propagation, by the following procedure:

- Calculate flux by integrating, up to $1150 \mathrm{~ns}$, the potential difference between the two HV capacitance plates which sandwich the ferrite of the cell of interest;

- Divide the flux by the current flowing through the terminating resistor at $\sim 1150 \mathrm{~ns}$; the current is calculated from the voltage drop across the resistor.

The same probe was used to measure the voltage of each capacitance plate and the voltage across the terminating resistor. The measurement data was processed to remove the DC offset of the channel of the oscilloscope, and then normalized to the average of the voltage values in the time range of $1000 \mathrm{~ns}$ to $1150 \mathrm{~ns}$ : this normalization corrects for any drift in the output voltage of the pulse generator. Fig. 4 shows both measured and predicted cell inductance.

The measured and predicted cell inductances are in good agreement for all the central cells (\#2 to \#10) of the kicker magnet: the average of the measured inductance, for 7 central cells (\#3 to \#9), is $62.2 \mathrm{nH}$, only $0.3 \%$ less than the predicted value. However the measured inductance of the end cells is approximately $30 \%$ greater than the predicted inductance. CERN have used the above procedure to measure the cell inductance of many kicker magnets and have developed an equation for end cell inductance: namely the effective length of the end cell is increased by an amount approximately equal to onequarter of the aperture height [9] (for the prototype KAON magnet the additional length was $21 \%$ of the aperture height and the measurement and predictions were 
in good agreement [3]). For the AGS magnet the additional effective cell length would be $20.25 \mathrm{~mm}$ $(81 \mathrm{~mm} / 4)$ i.e. $67 \%$ of the physical cell length: this gives an end cell inductance of $104 \mathrm{nH}$, which is close to the predicted inductance of $99.3 \mathrm{nH}$. The discrepancy between the measured and predicted end-cell inductance is not yet understood, but may be due to the prototype AGS magnet being tested without striplines (Fig. 1): further tests are needed to determine if this is the reason.

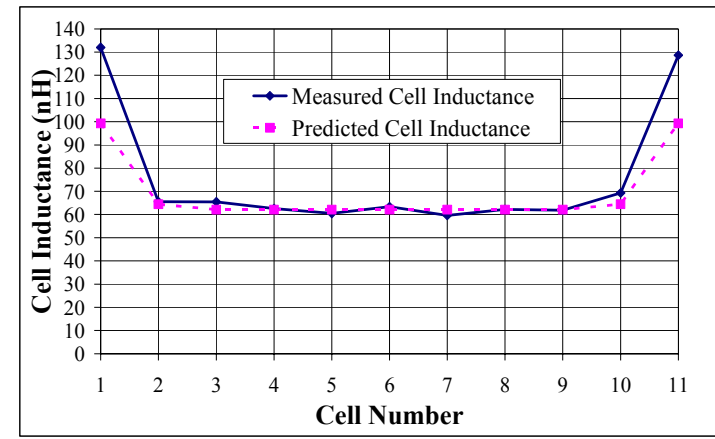

Figure 4: Measured and predicted cell inductance.

The measured propagation delay through the prototype A10 kicker magnet, at the $50 \%$ level, is $63 \mathrm{~ns}$ : the predicted propagation delay, for an end cell inductance of: (a) $99.3 \mathrm{nH}$ is $64.5 \mathrm{~ns}$, and (b) $130 \mathrm{nH}$ is $67.0 \mathrm{~ns}$. Thus the agreement between measured and predicted propagation delay indicates that the prototype A10 kicker end cell inductance is close to the theoretical value of $99.3 \mathrm{nH}$.

\section{Magnetic Field}

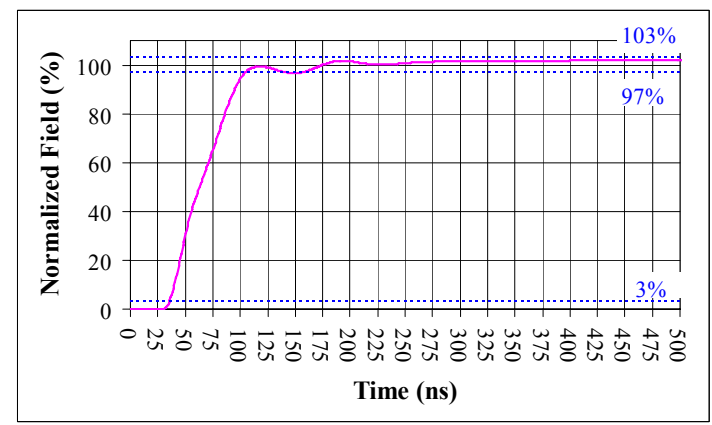

Figure 5: Measured magnetic field for prototype A10 kicker magnet (no peaking capacitors).

Fig. 5 shows the normalized field for the prototype A10 kicker magnet. The field is calculated from the integral, with respect to time, of the magnet input voltage minus the magnet output voltage normalized to the input voltage. Normalizing the output to the input voltage, before subtracting, compensates for losses in the busbars and also drift of the pulse generator. For the measurements the prototype kicker was not equipped with peaking capacitors, and hence there is no compensation for the end cell inductance being higher than that of a central cell. The rise-time of the magnetic field (Fig. 5), between 3\% and the first $97 \%$ crossing, is $69.5 \mathrm{~ns}$. The predicted 3\% to $97 \%$ magnetic field rise-time is:

- $\quad 68.3$ ns: no striplines or peaking capacitors, quality

factor of 10 for cell inductance, $99.3 \mathrm{nH}$ end cell 07 Accelerator Technology Main Systems inductance;

- 65.1 ns: no striplines or peaking capacitors, quality factor of 10 for cell inductance, $130 \mathrm{nH}$ end cell inductance;

- 95 ns: simulated A10 circuit, quality factor of 10 for cell inductance and $99.3 \mathrm{nH}$ end cell inductance.

The measured magnetic field rise-time is between $1.2 \mathrm{~ns}$ and $4.4 \mathrm{~ns}$ longer than predicted, however the risetime is within the $100 \mathrm{~ns}$ specification.

\section{CONCLUSIONS}

Capacitance measurements carried out on the prototype A10 kicker magnet are in excellent agreement with predictions. The measured and predicted cell inductances are in good agreement for all the central cells (\#2 to \#10) of the 11-cell kicker magnet. The average characteristic impedance of a central cell is $12.61 \Omega, 0.9 \%$ higher than the $12.5 \Omega$ goal: almost 0.4 percentage points of this error is due to the design value of the cell capacitance being $0.73 \%$ below the theoretical ideal value. The measured inductance of the A10 kicker end cells is approximately $30 \%$ greater than the predicted inductance. The $3 \mathrm{D}$ code used for the prediction of cell inductance has previously been shown to agree well with measurements [3]. In addition the predicted end cell inductance, for the prototype A10 kicker magnet, is in reasonable agreement with a rule of thumb, which is itself based on many measurements, developed at CERN. The magnetic field rise-time is within the $100 \mathrm{~ns}$ specification.

\section{REFERENCES}

1. R.B. Armenta, et al, "Design Concept for AGS Injection Kicker Upgrade to $2 \mathrm{GeV}$ ", Proc. PAC'05, May 16-20 2005, Tennessee, USA.

2. G.D. Wait, M.J. Barnes \& H.J. Tran, "Magnetic Field in a Prototype Kicker Magnet for the KAON Factory", IEEE Trans. Magnetics, July 1994, Vol. 30, No. 4, pp2118-2121.

3. M.J. Barnes \& G.D. Wait, "Comparison of Measured and Predicted Inductance per Cell for a Travelling Wave Kicker Magnet”, EPAC'96, Sitges, Spain, June 1996, pp2588-2590.

4. D. Fiander, K. Metzmacher \& P. Pearce, "Kickers and Septa at the PS Complex, CERN". Kaon PDS Magnet Design Workshop, Oct. 1988, pp71-79.

5. R. Armenta, M.J. Barnes, E. Blackmore, O. Hadary, D. Norn \& G.D. Wait, "Electromagnetic Modeling of the AGS A10 Injection Kicker Magnet", IEEE Trans. Superconductivity, June 2006, Vol. 16, issue 2, pp293-296.

6. Integrated Engineering Software, Winnipeg, Manitoba, Canada, www.integratedsoft.com.

7. D. Norn, "Computer Modeling of Kicker Magnets", TRIUMF Co-op Student Report, April 2005.

8. Fluke Test \& Measurement Equipment, www.fluke.com

9. Private communications with L. Sermeus, CERN. 